\section{Visual movement perception: A comparison of absolute and relative movement discrimination}

\author{
R. A. KINCHLA \\ Princeton University, Princeton, New Jersey 08540
}

It is proposed that there are two types of visual movement perception, absolute and relative. The former occurs when an object is seen to move in an otherwise homogeneous (or at least locally homogeneous) visual field. Relative judgments occur when one object is seen to move with respect to another, i.e., the separation between them is seen to change. Quantitative models for both processes are developed, and an experiment reported for which the models seem appropriate. The results appear relevant to a theory of size of length perception as well as to the general perceptual issue of absolute and relative judgments.

Earlier papers by this author and his colleagues (e.g., Kinchla \& Smyzer, 1967; Kinchla \& Allan, 1969, 1970) deal primarily with absolute movement perception: seeing an object move in an otherwise homogeneous visual field (Ganzfeld). This paper relates that perceptual process to another, termed relative movement perception: seeing one object move relative to the position of another object in your visual field. This distinction between absolute and relative movement perception is illustrated by the problem of judging the movement of a small cloud seen directly overhead in an otherwise clear blue sky. If its movement were difficult to discern, a natural strategy would be to move next to some object like a building so that the cloud appeared close to the top of the building. Movement of the cloud, originally imperceptable, is often quite apparent when seen in relation to the fixed reference point provided by the top of the building. The fact that two types of movement perception are involved is suggested by the illusion of "induced movement" (Dunker, 1929) occasionally produced in this situation: movement of the cloud "induces" an illusion that the building is moving ("falling forward or backwards") rather than the cloud. iote that one correctly perceives a change in separation between cloud and building (relative movement) but erroneously attributes it to (absolute) movement of the building.

A highly simplified form of the preceding perceptual situation was employed in the experiment reported in this paper. Rather than judging the movement of a cloud in a clear blue sky, Os were asked to judge the movement of a single point of light seen in the dark. Another point of light, rather than a

\footnotetext{
* The author is indebted to Charles Collyer for his assistance in conducting the experiment reported in this paper.
}

rooftop, was then introduced at various distances from the test light in order to assess the effect of a reference point on the Os' judgments.

Before considering the experiment, it will be useful to develop a more precise terminology with which to discuss visual movement perception, and to consider some simple theoretical models for the absolute and relative forms of this process.

\section{MOVEMENT DISCRIMINATION TASKS}

Perhaps the most elementary experimental situation one could employ to study absolute movement perception is to ask an $O$ to judge the movement of a single point of light presented in the dark. Two types of stimulus patterns one might employ are illustrated in Fig. 1. Illuminations of small points of light in an otherwise dark room are specified in time and space coordinates. Time in seconds is represented on the abscissa, while the ordinate denotes Lateral Position $L$ in degrees of visual angle. Position $0(L=0)$ corresponds to some arbitrary point in front of an $O$, and the other positions are specified by their horizontal angular displacement from Position 0, positive to the right and negative to the left. All points are assumed to be at an equal distance from the $O$. Two closely related types of movement patterns are illustrated: a discrete pattern, denoted by the solid lines, and a continuous pattern, denoted by a broken line. The continuous pattern consists of a point of light continuously illuminated from Time 0 until Time $t$, moving horizontally at a constant angular velocity of $v \mathrm{deg} / \mathrm{sec}$. The light comes on at Position 0 and goes off at Position $m$, so that the angular velocity, $v$, is simply $m$ divided by $\mathrm{t}$. The discrete pattern consists of successive illuminations of two stationary points of light; the first light is at Position 0 and the second at Position $m$, with a $t$-sec period of darkness between the successive illuminations. Thus both the discrete and continuous movement patterns in Fig. 1 can be defined in terms of $m$ and $t$. The exposure time, $e$, of the lights in the discrete pattern does not seem to be an important consideration, so long as it is sufficient to make the two stationary lights visible. The displacement, $\mathrm{m}$, will be referred to as the "movement of a pattern," with a "stationary" pattern simply one which has no movement $(\mathrm{m}=0)$.

Movement discrimination will be defined as an O's ability to discriminate movement patterns solely on the basis of movement. Thus, movement discrimination is required to distinguish any pair of movement patterns, $S_{i}$ and $S_{j}$, which are identical except for their respective $m$ values, $m_{i}$ and $m_{j}$. If $S_{i}$ and $S_{j}$ differ in any other respect, it is difficult to determine whether the $O$ is really basing his discrimination on the $m$ values or on this other dimension. For example, if $S_{i}$ and $S_{j}$ differ in respect to $t$, the $\mathrm{O}$ might simply make a temporal discrimination. It should be emphasized that discriminating a discrete pattern from a continuous pattern is not movement discrimination. The familiar phi illusion is not an error in movement discrimination (the two successively illuminated lights actually are in different positions); rather, it is a failure to discriminate between discrete and continuous movement. Thus, "movement perception" as employed here will simply denote the perception of $a$ change in position.

The subsequent theoretical arguments are meant to apply to either discrete or continuous movement patterns, although only discrete patterns were employed in the experiment reported in this paper. In either case, the theoretical arguments can be developed most simply in terms of a simple form of movement discrimination: distinguishing a stationary point of light from one that moves to the right. These two stimulus patterns will be denoted,

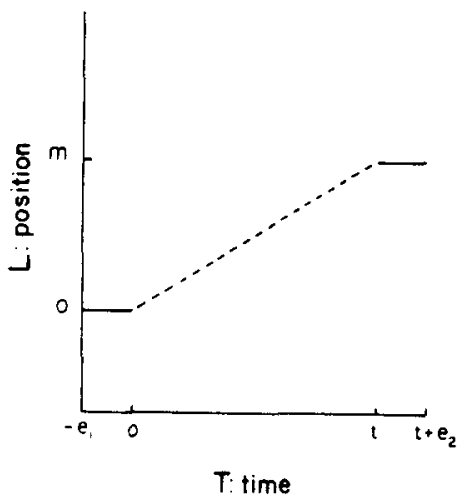

Fig. 1. An illustrative $S_{i}$ discrete (solid lines) and continuous (broken line) movement stimulus pattern. 


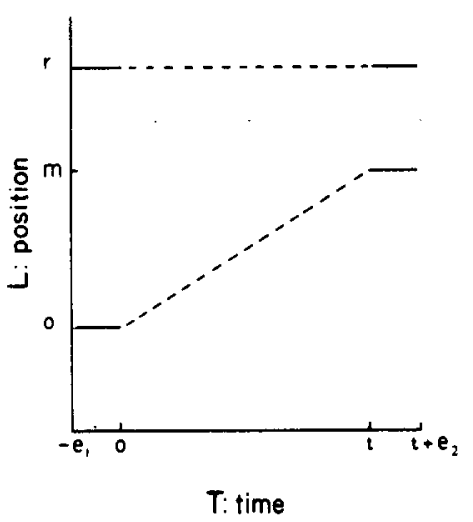

Fig. 2. An illustrative $S_{i}$ discrete (solid lines) and continuous (broken line) movement pattern with a reference light at Position r.

respectively, by $S_{0}$ and $S_{1}$, where the movement of the stationary pattern, $m_{0}$, equals zero and the movement of the $S_{1}$ pattern, $m_{1}$, is greater than zero; i.e., the second light is displaced to the right $m_{1}$ deg. The discrimination task consists of a series of trials, with either $S_{1}$ or $S_{0}$ presented with equal probability on each trial. Following each presentation of a pattern the $O$ must indicate either that $S_{1}$ was presented, an $R_{1}$ response, or that $S_{0}$ was presented, an $\mathrm{R}_{0}$ response.

In order to perceive the movement defined by one of the patterns (discrete or continuous) in Fig. 1, the $O$ must recognize that the position of the light at Time $t$ is different from the position of the light at Time 0 In this sense his perceptual problem is one of visual position memory. A model of perceptual memory (Kinchla \& Smyzer, 1967) originally employed to represent an $O$ 's memory for auditory amplitude, has been successfully developed as a model for discriminations involving patterns of the type illustrated in Fig. 1 (Kinchla \& Allen, 1969). However, consider the type of visual position memory that might be employed to perceive the movement of the pattern illustrated in Fig. 2. Here a reference light at Position $r$ is added to the patterns illustrated in Fig. 1. In a discrete pattern, this reference light is illuminated in synchrony with the two successively illuminated stationary points, while in the continuous case, the reference point is illuminated continuously from Time 0 to Time $t$. If the $O$ were to ignore the reference point, his perceptual problem would be the same as before, he must "remember" the absolute location of Position 0 until Time $t$ in order to notice the movement, $\mathrm{m}$. However, the $\mathrm{O}$ might simply "remember" the relative position of the two lights at Time 0 (the distance between Position 0 and Position r) and compare this memory with the separation between the lights at Time $t$ (the distance between Position $m$ and Position r). If his memory for relative position were more efficient than his memory for absolute position, he would be more accurate in discriminating movement when the reference light was present.

\section{A MODEL FOR ABSOLUTE MOVEMENT PERCEPTION}

The model for absolute movement perception (Kinchla \& Allan, 1969) may be summarized as follows. Let $S_{i}$ be a stimulus pattern of the sort illustrated in Fig. 1, with Parameters $m_{i}$ and $t$. Each presentation of $S_{i}$ produces some value $x_{A}$ of a hypothetical variable $X_{A}$, where $x_{A}$ equals the actual movement of the pattern $\left(m_{i}\right)$ plus a Gaussian random variable, $N_{A}$. This "noise" variable has an expected value of zero and a variance defined as follows:

$$
\operatorname{Var}\left(N_{A}\right)=\phi_{A} t+2 \pi_{A}
$$

The constants $\phi_{A}$ and $\pi_{A}$ can be interpreted as measures of the "noise" in different phases of the perceptual process. Specifically, $2 \pi_{A}$ denotes the total input noise accrued in generating internal representations of the initial and terminal positions of the light, while $\phi_{\mathrm{A}} \mathrm{t}$ is the memory noise accrued in maintaining a memory of the initial position during the $t_{i}$ sec before the terminal position is defined (i.e., memory noise is directly proportional to $t$. The expected value and variance of $X$ will be related to the stimulus parameters as follows:

$$
\begin{aligned}
\mathrm{E}\left(\mathrm{X} \mid \mathrm{S}_{\mathrm{i}}\right) & =m_{\mathrm{i}}+\mathrm{E}\left(\mathrm{N}_{\mathrm{A}}\right) \\
& =m_{\mathrm{i}} ;
\end{aligned}
$$

and

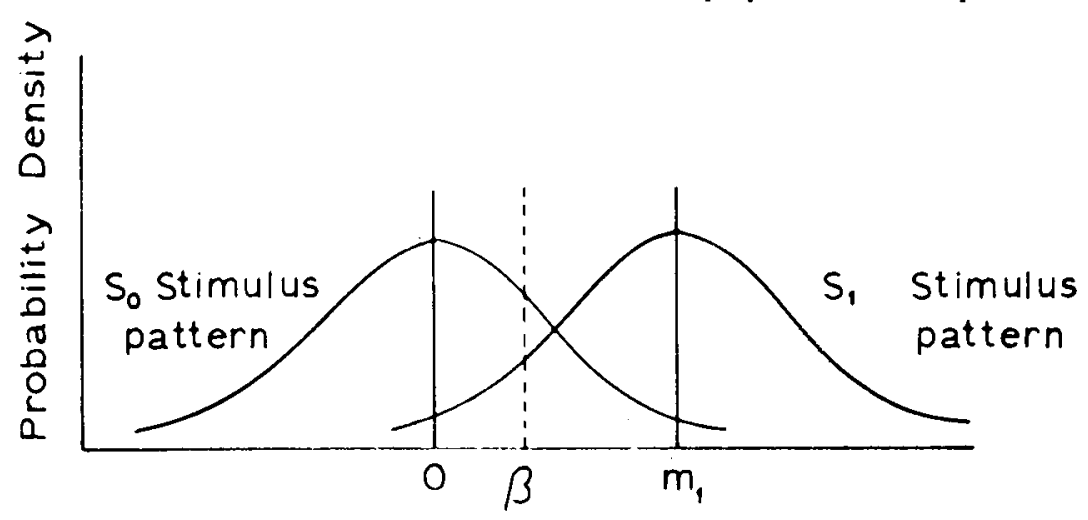

\section{$X$ : Subjective impression of movement}

Fig. 3. Hypothetical distributions of $X_{A}$ (or $X_{R}$ ) given $S_{0}$ and $S_{1}$ with an arbitrary response criterion. 
$O$ made to an $S_{i}$ pattern $(i=0,1)$, and $\hat{L}_{i}$ denotes that value of a normal deviate exceeded with a probability equal to $\hat{\mathrm{r}}\left(R_{1} \mid S_{\mathrm{i}}\right)$, then the difference between $\hat{\iota}_{0}$ and $\hat{z}_{1}$ is an estimate of $\hat{\mathrm{V}}_{\mathrm{A}}$, denoted $\mathrm{D}_{\mathrm{A}}$ :

$$
\hat{\mathrm{D}}_{\mathrm{A}}=\hat{z}_{0}-\hat{\mathrm{L}}_{1} \text {. }
$$

It has been shown (Kinchla \& Allan, 1969) that in most applications of this model the input noise $\left(2 \pi_{\mathrm{A}}\right.$ in Eq. 3 ) is negligible relative to the memory noise $\left(\dot{\varphi}_{A} t_{i}\right.$ in Eq. 3). While there may be situations in which input noise could be an important component (e.g., if the $O$ were seated on a vibrating platform), the general model can usually be defined in a one-parameter form $\left(\pi_{A}=0\right)$ so that Eq. 4 becomes

$$
\dot{\mathrm{D}}_{\mathrm{A}}=\frac{\mathrm{m}_{1}}{\left(\phi_{\mathrm{A}} t\right)^{1 / 2}}
$$

with $\phi_{\mathrm{A}}$ equal to about $1.8 \mathrm{sq} \mathrm{deg} / \mathrm{sec}$ for a typical 0.

\section{A MODEL FOR \\ RELATIVE MOVEMENT PERCEPTION}

It is proposed here that an $O$ may also "input" and "remember" the separation between two lights, although the efficiency of this process is inversely related to the size of the separation. In other words, it is proposed that a model for relative judgments can be defined in a manner analogous to that for absolute judgments, except that the noise in both the input and memory processes will be proportional to the separation between the two lights, i.e., a type of Weber assumption. Specifically, let $S_{i}$ be a pattern of the sort illustrated in Fig. 2 and assume that each presentation of $S_{i}$ evokes a value $x_{R}$ of a hypothetical variable $X_{R}$, where $x_{R}$ equals the actual movement of the pattern $\left(\mathrm{m}_{\mathrm{i}}\right)$ plus a Gaussian random variable, $N_{R}$. This noise variable has an expected value of zero and a variance defined as follows:

$\operatorname{Var}\left(N_{R} \mid S_{i}\right)=\left(\phi_{R} t+\pi_{R}\right) r+\pi_{R}\left(r-m_{i}\right)$

Note that quantity $\left(\phi_{R} t+\pi_{R}\right) r$ can be interpreted as the noise accrued when the initial separation between the two lights ( $r$ ) is input and remeinbered until Time t. Whereas the quantity $\pi_{R}\left(r-m_{i}\right)$ is simply the noise accrued when the terminal separation between the two lights $\left(r \cdots m_{i}\right.$ is input at Time $\mathrm{t}$.

The expected value and variance of $X_{R}$ will be defined as follows:

$$
\begin{aligned}
E\left(X_{R} \mid S_{i}\right) & =m_{i}+E(N) \\
& =m_{i}
\end{aligned}
$$

and

$$
\begin{aligned}
\operatorname{Var}\left(X_{R} \mid S_{i}\right) & =\operatorname{Var}\left(N \mid S_{i}\right) \\
& =\left(\phi_{R} t+\pi_{R}\right) r+\pi_{R}\left(r-m_{i}\right) .
\end{aligned}
$$

Assuming that an $\mathrm{O}$ reports relative movement (reports $S_{1}$ ) only when $x_{R}$ exceeds some criterion for relative movement, $\beta_{\mathrm{R}}$, his decision problem is essentially the same as that encountered in absolute judgments (and illustrated in Fig. 3). The model is slightly more complicated since, by Eq. 9 ,

$$
\operatorname{Var}\left(X_{R} \mid S_{0}\right)=\left(\phi_{R} t+2 \pi_{R}\right) r
$$

while

$\operatorname{Var}\left(X_{R} \mid S_{1}\right)=\left(\phi_{R} t_{i}+\pi_{R}\right) r+\pi_{R}\left(r-m_{1}\right)$

Thus while $\operatorname{Var}\left(X_{A} \mid S_{0}\right)$ equals $\operatorname{Var}\left(X_{A} \mid S_{t}\right), \quad \operatorname{Var}\left(X_{R} \mid S_{0}\right)$ exceeds $\operatorname{Var}\left(X_{R} \mid S_{1}\right)$ by the amount $m_{1} \pi_{R}$.

A discriminability measure for relative judgments, $D_{R}$, can be defined in a manner analogous to $D_{A}$ (Eq. 4):

$$
\begin{aligned}
D_{R} & =\frac{E\left(X_{R} \mid S_{1}\right)-E\left(X_{R} \mid S_{0}\right)}{\left[\operatorname{Var}\left(X_{R} \mid S_{0}\right)\right]^{1 / 2}} \\
& =\frac{m_{1}}{\left[\left(\phi_{R} t+2 \pi_{R}\right) r\right]^{1 / 2}} .
\end{aligned}
$$

However, because $\operatorname{Var}\left(X_{R} \mid S_{0}\right)$ does not equal $\operatorname{Var}\left(X_{R} \mid S_{1}\right)$, our estimate of $D_{R}$ differs slightly from that in the absolute model (Eq. 5):

$$
\hat{D}_{\mathrm{R}}=\hat{z}_{0}-k \hat{\grave{L}}_{1},
$$

where

$$
k=\frac{\left[\operatorname{Var}\left(X_{R} \mid S_{1}\right)\right]^{1 / 2}}{\left[\operatorname{Var}\left(X_{R} \mid S_{0}\right)\right]^{1 / 2}}
$$

It should be noted that $k$ is approximately one if the quantity $\pi_{R} m_{1}$ is small with respect to the quantity $\phi_{R} t r+2 \pi_{R} r$ (see Eq. 11). When $k$ is close to one, an estimate of $D_{R}$ can be obtained in the same fashion as $D_{A}$ in Eq. 5 , i.e.,

$$
\hat{\mathrm{D}}_{\mathrm{R}} \cong \dot{\mathrm{z}}_{0}-\hat{\mathrm{L}}_{1} \text {. }
$$

The preceding assumptions imply that relative movement judgments will be superior to absolute judgments only if the "noise" in the former process, $\operatorname{Var}\left(X_{R} \mid S_{0}\right)$, is less than that in the latter, $\operatorname{Var}\left(\mathrm{X}_{\mathrm{A}}\right)$; specifically, $\mathrm{D}_{\mathrm{R}}$ in Eq. 12 is greater than $D_{A}$ in $E q .6$ only if $\left(\phi_{R} t+2 \pi_{R}\right) r$ is less than $\phi_{A} t$. It is also clear from Eq. 12 that $D_{R}$ diminishes as $r$ increases. Thus, if $r_{c}$ is a value of $r$ such that $\phi_{A} t$ equals $\left(\phi_{R} t+2 \pi_{R}\right) r_{c}$, or

$$
r_{c}=\frac{\phi_{A} t}{\phi_{R} t+2 \pi_{R}},
$$

then an $\mathrm{O}$ might make relative judgments only when $r$ is less than $r_{c}$. If $r$ is greater than $r_{c}$, he can do better if he ignores the reference light and simply makes an absolute judgment. This argument suggests that the discriminability of patterns like those in Fig. 1b should initially diminish as $r$ is increased (by Eq. 12) until discrimination is equal to that attained without a reference light (Eq. 4); performance should then be unaffected by further increments in $r$. (One might also assume that $N_{A}$ and $N_{R}$ are at least partially independent and that the $O$ could use both $X_{A}$ and $X_{R}$ in each judgment. While models of this sort are interesting, the simpler assumption that either $\mathrm{X}_{\mathrm{A}}$ or $X_{R}$ is used will be employed in the present analysis.)

\section{AN EXPERIMENT}

The following experiment was designed to assess the role of a reference light in movement perception. Three Os performed a discrete movement discrimination task involving two stimulus patterns, $S_{0}$ and $S_{1}$, with $m_{0}$ equal to 0 deg and $m_{1}$ equal to $.13 \mathrm{deg}$. Sometimes a reference light was presented, as in Fig. 2, with $\mathrm{r}$ equalling 1, $3,5,10,15$, or $20 \mathrm{deg}$, while other times there was no reference light, as in Fig. 1. In all cases, the interval $t$ was either .5 or 2 sec. (These particular values were chosen on the basis of preliminary testing with other Os.) The possible combinations of these factors defined 14 experimental conditions.

\section{Procedure}

Each $\mathrm{O}$ was tested daily for 35 days. The first seven sessions were considered practice, with each of the 14 conditions in effect during one-half of a particular session. A single condition was in effect throughout each of the remaining 28 sessions, such that two sessions were run under each of the 14 conditions. The particular sequence of conditions was determined randomly for each $\mathbf{O}$. 
Table 1

Values of $\hat{\mathbf{P}}\left(R_{1} \mid S_{1}\right)$, Denoted $\hat{\mathbf{P}}_{1}, \hat{\mathbf{P}}\left(R_{1} \mid S_{0}\right)$, Denoted $\hat{\boldsymbol{P}}_{0}$, and $\hat{\vec{P}}_{c}$, for Each Observer Under Each Experimental Condition. The no-reference-light absolute-judgment condition is denoted by $\mathbf{r}=\mathbf{A}$.

\begin{tabular}{|c|c|c|c|c|c|c|c|c|c|c|}
\hline \multirow[b]{2}{*}{$\begin{array}{c}t \\
\text { (Seconds) }\end{array}$} & \multirow[b]{2}{*}{$\mathbf{r}$} & \multicolumn{3}{|c|}{01} & \multicolumn{3}{|c|}{02} & \multicolumn{3}{|c|}{03} \\
\hline & & $\hat{p}_{1}$ & $\hat{\mathbf{P}}_{0}$ & $\hat{\mathbf{P}}_{\mathrm{c}}$ & $\hat{\mathrm{P}}_{1}$ & $\hat{\mathbf{P}}_{0}$ & $\hat{\mathbf{P}}_{\mathrm{c}}$ & $\hat{\mathbf{P}}_{1}$ & $\hat{\mathbf{P}}_{0}$ & $\hat{\mathbf{P}}_{\mathrm{C}}$ \\
\hline .5 & $1 \mathrm{Deg}$ & .74 & .27 & .74 & .72 & .14 & .79 & .63 & .29 & .67 \\
\hline .5 & $3 \mathrm{Deg}$ & .63 & .32 & .66 & .47 & .15 & .66 & .63 & .46 & .58 \\
\hline .5 & $5 \mathrm{Deg}$ & .56 & .33 & .62 & .61 & .31 & .65 & .63 & .47 & .58 \\
\hline .5 & $10 \mathrm{Deg}$ & .52 & .38 & .57 & .61 & .39 & .61 & .56 & .38 & .59 \\
\hline .5 & $15 \mathrm{Deg}$ & .64 & .45 & .60 & .41 & .21 & .60 & .57 & .41 & .58 \\
\hline .5 & $20 \mathrm{Deg}$ & .46 & .25 & .60 & .57 & .35 & .61 & .51 & .39 & .56 \\
\hline .5 & A & .38 & .21 & .58 & .67 & .45 & .61 & .47 & .32 & .58 \\
\hline 2 & $1 \mathrm{Deg}$ & .61 & .34 & .64 & .47 & .16 & .66 & .63 & .42 & .60 \\
\hline 2 & $3 \mathrm{Deg}$ & .59 & .40 & .60 & .43 & .23 & .60 & .61 & .50 & .56 \\
\hline 2 & $5 \mathrm{Deg}$ & .48 & .34 & .57 & .42 & .27 & .58 & .51 & .46 & .52 \\
\hline 2 & $10 \mathrm{Deg}$ & .47 & .35 & .56 & .62 & .47 & .58 & .50 & .46 & .52 \\
\hline 2 & $15 \mathrm{Deg}$ & .47 & .37 & .55 & .40 & .30 & .55 & .46 & .36 & .55 \\
\hline 2 & $20 \mathrm{Deg}$ & .40 & .30 & .55 & .48 & .35 & .56 & .46 & .38 & .54 \\
\hline 2 & A & .41 & .31 & .55 & .54 & .43 & .56 & .38 & .32 & .53 \\
\hline
\end{tabular}

A daily testing session was divided into five successive blocks of 100 trials, with a $1-$ min rest period in the dark between blocks. Each trial began with a clearly audible 1 -sec-duration $500-\mathrm{Hz}$ warning tone, followed immediately by the stimulus pattern. The $\mathrm{O}$ then had $2 \mathrm{sec}$ in which to indicate his response (pressing one of two buttons) before the start of the next trial. Both $S_{1}$ and $S_{0}$ patterns occurred equally often in a randomly determined sequence within each block of trials.

The stimulus lights were tungsten filament "indicator" lamps (Dialco No. 39) with a flat circular surface of white opal glass. They were equated photometrically and interchanged several times during the experiment. They were located $4.1 \mathrm{~m}$ in front of the $O$ where they subtended $.033 \mathrm{deg}$ of visual angle. The lights were clearly visible at about $4 \mathrm{~mL}$, although not bright enough to reveal any other details in the otherwise dark testing room. Each illumination of a light was for $100 \mathrm{msec}$ $\left(e_{1}\right.$ and $e_{2}$ in Figs. 1 and 2).

\section{Results}

The first 100 trials during each of the 28 data sessions were treated as "warmup" or practice trials, so that each $\mathrm{O}$ produced 800 trials of data (400 for each stimulus) under each of the 14 experimental conditions. His performance under each condition will be summarized by the proportion of $R_{1}$ responses to each stimulus, $\hat{\mathrm{P}}\left(R_{1} \mid S_{1}\right)$ and $\hat{P}\left(R_{1} \mid S_{0}\right)$. It is also interesting to consider the proportion of "correct" responses denoted by $\hat{\mathrm{P}}_{c}$, where

$$
\hat{\mathrm{P}}_{\mathrm{c}}=.5 \hat{\mathrm{P}}\left(\mathrm{R}_{1} \mid \mathrm{S}_{1}\right)+.5\left[1-\hat{\mathrm{P}}\left(\mathrm{R}_{1} \mid \mathrm{S}_{0}\right)\right] .
$$

These proportions for each $\mathrm{O}$ under each experimental condition are presented numerically in Table 1 , with each proportion based on 400 trials of data.

be employed in

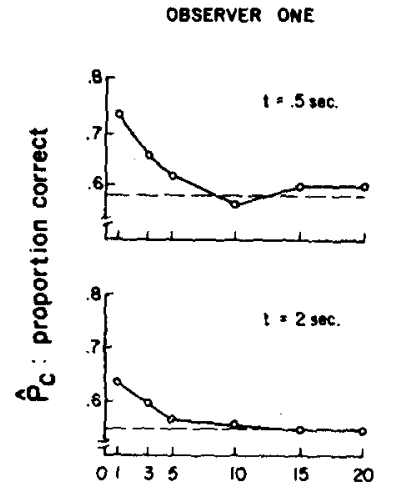
points) and without (broken lines) a reference light. obtained with a reference light (Eq. 15). This procedure seems appropriate for two reasons: First, the Os may have made absolute judgments even when the reference light was presented, and second, it will be shown that Eq. 15 seems an appropriate approximation ( $\mathrm{k}$ in Eq. 16 is approximately one) even when the 0 appears to have made a relative judgment. (The reader who wishes to apply the model without making this assumption is referred to the section in Green \& Swets, 1968, which discusses the "unequal variance" model of signal detection.) Since $\hat{\mathrm{D}}_{\mathrm{A}}$ and $\hat{D}_{\mathrm{R}}$ are both defined as the difference between $\hat{z}_{0}$ and $\hat{z}_{1}$ (Eqs. 5 and 15), the discriminability measure will simply be denoted by $\hat{D}$. This measure, for each $\mathrm{O}$ in each experimental condition, is presented numerically in Table 2 and graphically in Fig. 5.

First of all, consider the values of $\hat{D}$ obtained without a reference light. These measures are presented in the bottom row (labeled "A" for absolute) in Table 2, and as solid data points at the far right of each graph in Fig. 5. Previous work (e.g., Kinchla \& Allan, 1969) has shown that absolute judgments of this kind are consistent with the one-parameter form of the absolute movement perception model. Accordingly, an estimate of $\phi_{A}$ was obtained for each $\mathrm{O}$ by selecting that value of $\hat{\phi}_{A}$ which minimized the sum of squared deviations between the values of $D$ he attained without a reference light, with $t=.5 \mathrm{sec}$ and $t=2 \mathrm{sec}$, and those defined by Eq. 6 . These $\hat{\phi}_{\mathrm{A}}$ values were $.120, .132$, and $.180 \mathrm{sq} \mathrm{deg} / \mathrm{sec}$ for $O s \mathrm{~s}, 2$, and 3, respectively. The theoretical values of $D$ for each $\hat{\phi}_{A}$ value are indicated by horizontal broken lines on each graph in Fig, 5; the upper line indicates the predicted $D$ for $t=.5 \mathrm{sec}$ and the lower line for $t=2 \mathrm{sec}$. It is clear that the superior discriminability without a

OBSERVER TWO

OBSERVER THAEE
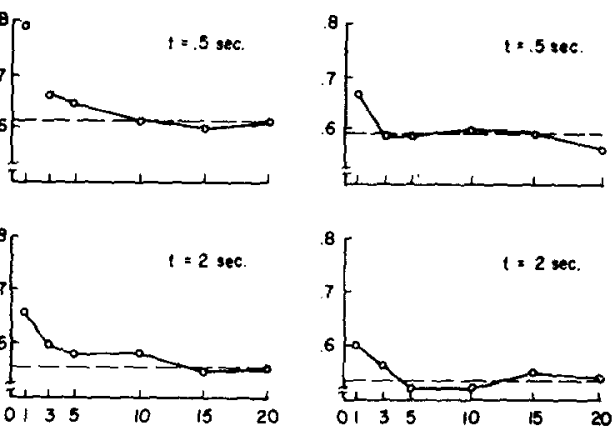

$\mathbf{r}$ : proximity of reference light in degrees visual angle

Fig. 4. Values of $\hat{P}_{c}$ for each $O$ under each experimental condition both with (data 

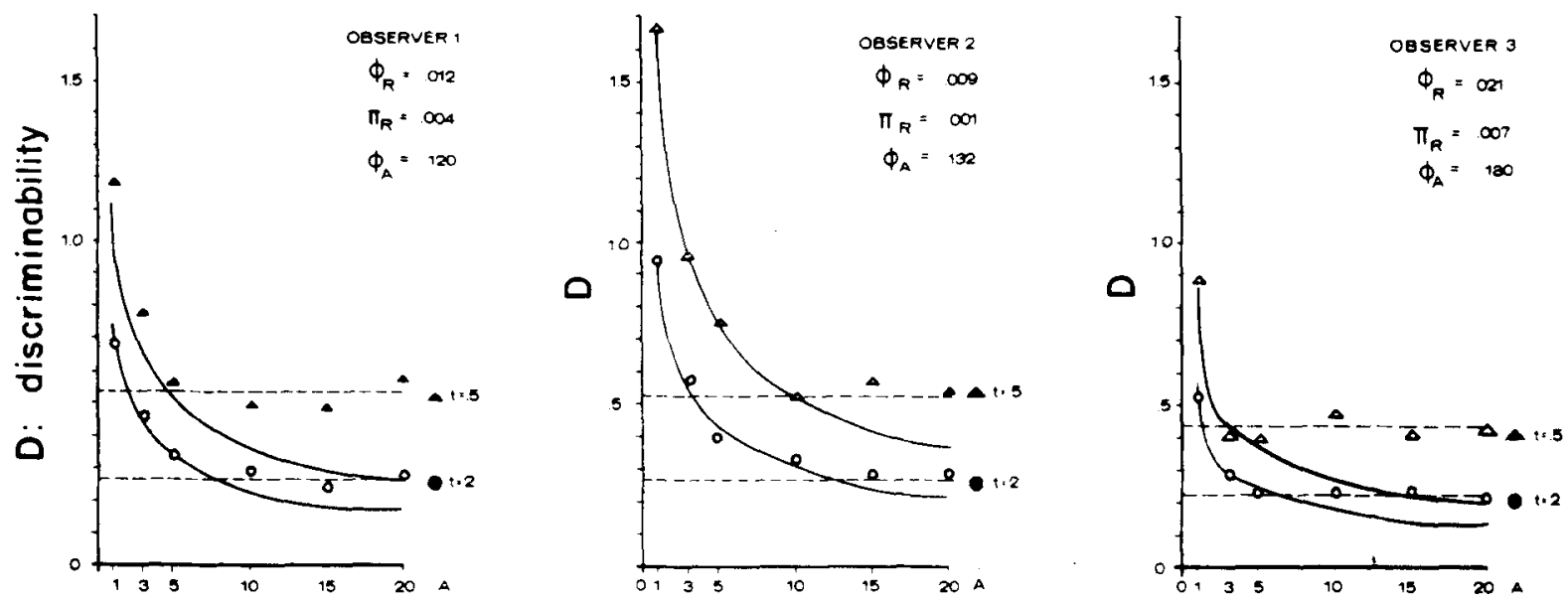

\section{$r$ : proximity of reference light in degrees visual angle}

Fig. 5. Estimated (data points) and theoretical values of $D_{A}$ and $D_{R}$ for each $O$ in each experimental condition.

reference light at the larger $(2 \mathrm{sec}) \mathrm{t}$ value is generally consistent with the absolute judgment model. Furthermore, the values of $\hat{\phi}_{\mathrm{A}}$ are similar to those obtained in earlier studies of absolute-movement perception.

The fact that a reference light improved performance only when $r$ was small is as apparent in Fig. 5 as it was in Fig. 3. The chief advantage of considering $D$ rather than $\hat{\mathrm{P}}_{\mathrm{c}}$ is that the former is theoretically independent of response bias $(\beta)$, whereas $\hat{\mathrm{P}}_{\mathrm{c}}$ is not. However, Eq. 12 specifies the theoretical relation between $\mathrm{m}, \mathrm{t}$, and $\mathrm{r}$ (the independent variables) and $\hat{D}$ (the dependent variable) only when the $O$ was making a relative judgment. It was proposed earlier in this paper that an $O$ makes relative judgments only when they are superior to absolute judgments, i.e., when $D_{R}>D_{A}$. Thus, the relative judgment model would apply only in those experimental conditions where the $O$ did better than could be attributed to the absolute judgment process. While more elaborate strategies for identifying licse conditions could be derived, the following simple procedure seemed suitable for purposes of this paper: It was assumed that an $\mathrm{O}$ utilized relative judgments at all $\mathrm{r}$

Table 2

Values of $D$ for Each $O$ in Each Experimental Condition Based on the Data in Table 1 and Eq. 5

\begin{tabular}{|c|c|c|c|c|c|c|}
\hline \multirow[b]{2}{*}{$\mathbf{I}$} & \multicolumn{2}{|c|}{01} & \multicolumn{2}{|c|}{$\mathrm{O} 2$} & \multicolumn{2}{|c|}{$\mathrm{O} 3$} \\
\hline & $\mathrm{t}=.5$ & $t=2.0$ & $\mathrm{t}=.5$ & $t=2.0$ & $\mathrm{t}=.5$ & $\mathrm{t}=2.0$ \\
\hline I & 1.18 & .68 & 1.67 & 94 & .88 & .53 \\
\hline 3 & .77 & .46 & .96 & .58 & 40 & .28 \\
\hline 5 & .57 & .34 & .76 & .40 & .39 & .24 \\
\hline 10 & .49 & .29 & .53 & .34 & .47 & .24 \\
\hline 15 & .48 & .25 & .58 & .28 & .40 & .25 \\
\hline 20 & .57 & .28 & .55 & .29 & .42 & .22 \\
\hline A & .52 & .26 & .54 & .26 & .40 & .20 \\
\hline
\end{tabular}

values lower than the smallest $r$ condition for which $\dot{D}$ was smaller than that predicted by the absolute judgment model. For example, for $\mathrm{O} 1$ this included the $\mathrm{r}=1,3$, and $5 \mathrm{deg}$ conditions when $t=.5 \mathrm{sec}$, and the $r=1,3,5$, and $10 \mathrm{deg}$ conditions when $t=2 \mathrm{sec}$. Estimates of $\phi_{R}$ and $\pi_{R}$ were then obtained which minimized the sum of squared discrepancies between the $\dot{D}$ values for those seven conditions and those defined by Eq. 12. Estimates for the other two Os were calculated in a similar fashion. The values of $\dot{\phi}_{\mathrm{R}}$ for $\mathrm{O}_{\mathrm{S}} 1,2$, and 3 , respectively, were .012, .009, and .021, while the corresponding values of $\hat{\pi}_{\mathrm{R}}$ were $.004, .001$, and .007 . The theoretical values of $D_{R}$ defined by these estimates and Eq. 12 are shown by the continuous curves on each graph in Fig. 5. To be consistent with the earlier arguments the data points should lie on the solid curve so long as it is above the broken line and on the broken line otherwise.

It is clear from inspection of Fig. 5 that the theoretical arguments provide a plausible interpretation of the data. Discriminability (i) seems consistent with the relative judgment process so long as $\mathrm{D}_{\mathrm{R}}>\mathrm{D}_{\mathrm{A}}$, and consistent with the absolute judgment process when $\mathrm{D}_{\mathrm{R}}<\mathrm{D}_{\mathrm{A}}$. For examplc, consider the $\mathrm{t}=.5$ conditions for O 1. The relative judgment curve (solid line) drops below the absolute judgment curve (broken line) at $r=4.3 \mathrm{deg}\left(\mathrm{r}_{\mathrm{c}}\right.$ in Eq. 16). The $\hat{D}$ values for $r<4.3 \mathrm{deg}$ are consistent with the relative judgment curve, whereas the other $\hat{D}$ values are generally consistent with the absolute judgment model (i.e., consistent with performance when no reference light was presented). Similar features can be seen in the data obtained when $t=2 \mathrm{sec}$, and in the data from the other two Os. In each case, the data suggest a shift from relative to absolute judgments when $r$ exceeds $r_{c}$; $r_{c}$, Eq. 16, is indicated by the point at which the theoretical discriminability functions for absolute and relative judgments intersect in Fig. 5.

Note that a positive relation between $r_{c}$ and $t$ follows from Eq. 16 when $\phi_{A}>\phi_{R}$, and $\hat{\phi}_{A}$ exceeded $\hat{\phi}_{R}$ for all three Os. Thus the results suggest that the minimal proximity of reference light required to produce relative judgments is positively related to $t$. However, Eq. 16 implies that $r_{c}$ approaches a limiting value equal to $\phi_{\mathrm{A}} / \dot{\phi}_{\mathrm{R}}$ as $\mathrm{t}$ becomes very large. The largest limiting value of $r_{c}$ is suggested by the performance of $\mathrm{O} 2$, whose theoretical parameter estimates indicate a limit of $14.3 \mathrm{deg}$. Thus, even if $t$ was much larger, all of the Os would be expected to have reverted to absolute judgments when the reference light was more than about $15 \mathrm{deg}$ from the test light.

It should be emphasized that the preceding analysis is not presented as an extensive empirical test of the relative judgment model. The model is applied here in a highly tentative fashion, both to illustrate its application and to provide an explicit quantitative framework in which to discuss the data. Nevertheless, the two models seem to provide a reasonable interpretation of the experimental results.

\section{DISCUSSION}

The experimental evidence appcars consistent with a two-process view of visual movement perception. Os seem to make absolute judgments not only when an object is seen in an otherwise totally homogeneous visual field (Ganzfeld). but also when the field is only locally devoid of reference points (a "local Ganzfeld"). The minimal proximity of a reference point 
(a)

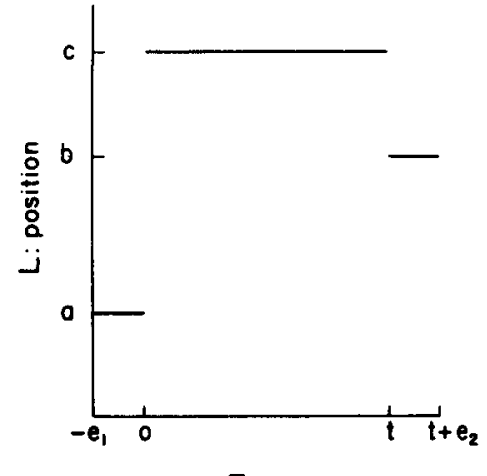

T: time (b)

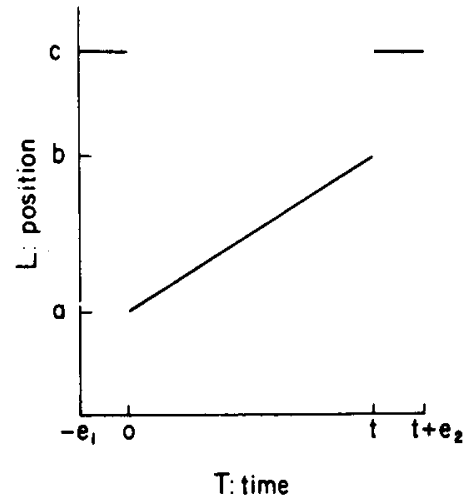

Fig. 6. Alternatives to the type of stimulus patterns shown in Fig. 2 which might also produce relative judgments.

which will produce relative judgments is, apparently, never much more than $15 \mathrm{deg}$, although the point must be even closer if the stimulus is only briefly observed $(\mathrm{t}<2$ of $3 \mathrm{sec}$ ). This minimal proximity, $r_{\mathrm{c}}$, is specified by Eq. 16. Furthermore, while the present experiment utilized discrete movement patterns, the conclusions should also apply to fixed-velocity continuous movement since $r_{c}$ is independent of $m$; i.e., if an object were moving continuously at a fixed velocity, $v$ (equal to $m / t$ ), the value of $r_{\mathfrak{c}}$ should depend only on the period of observation, $t$.

While the present analysis suggests that an 0 uses either absolute or relative judgments, it is possible that he can utilize both at the same time, and it would be premature to conclude that the two types of judgmental processes are mutually exclusive simply on the basis of the data presented here. However, the simple mutually exclusive assumption does seem to provide a reasonably satisfactory interpretation of these data.

The phenomenon of induced movement is a natural consequence of the two theoretical processes. If two objects are sufficiently close, one might clearly discern relative movement while having little basis for an absolute judgment. This would be the case whenever $D_{R}$ was large while $D_{A}$ was very small, for example in the $r=1 \mathrm{deg}$ condition in the present experiment. However, here the Os knew that the right-hand light in each display was always in the same position (Position r). Thus they could always interpret a perception of relative movement as indicating a displacement of the light on the left. If an $O$ did not know which of the two lights might be displaced, he could be quite accurate in discriminating a change in the separation between the two lights (relative movement) while essentially having to guess which light had actually been displaced (absolute movement). Experiments of this sort have been conducted in this laboratory and are in preparation for publication. While a detailed development of this work will not be attempted here, it indicates that the clarity of relative movement increases as the two lights are brought closer together, almost independently of the O's ability to discern which light has actually moved.

How relative movement is interpreted in the absence of an unambiguous reference point is particularly interesting. In general, it seems as if an $O$ simply adopts one point as his reference and interprets all movement in respect to it. For example, a common procedure for demonstrating the illusion of induced movement is to project a small spot of light on a large screen. If the spot remains stabile, an 0 will often report that it was the spot that moved; i.e., he correctly perceives the relative movement of the spot with respect to the screen, but mistakenly interprets this as movement of the spot. This suggests that an $O$ tends to choose the larger of two objects as a reference point. Conjectures of this sort would seem amenable to an experimental analysis based on the theoretical approach developed in this paper.

Finally, it should be noted that there are some obvious alternatives to the types of stimulus patterns represented in Fig. 2. For example, suppose the reference point was presented only during the period from Time 0 to Time $\mathbf{t}$, as shown in Fig. 6a. An $O$ could either make a relative judgnent by comparing the separation defined at Time $0, c-a$, to that defined at Time $t$, $c-b$, or make an absolute judgment by comparing the position defined prior to Time 0 , a, with that defined after Time $t$, b. Similarly, movement defined as in Fig. $6 b$ could, also produce either an absolute or relative judgment. In any case, the theoretical models seem appropriate even if patterns of the type shown in Fig. 6 (rather than Fig. 2) had been employed in the screen is surreptitiously moved while the experiment. The stimulus parameters would still be $\mathrm{m}, \mathrm{t}$, and $\mathrm{r}$, and there is no obvious reason to suppose the theoretical ( $\phi$ and $\pi$ ) parameters would differ. Thus, in the absence of further data, it seems reasonable to suppose that our general conclusions apply to movement patterns of this sort as well.

\section{LENGTH PERCEPTION}

One could interpret the preceding experiment as involving judgments of length: The Os were asked to compare a separation ("length") presented at Time 0 with one presented $t \mathrm{sec}$ later. Thus, the theoretical arguments should also be relevant to a theory of length (or "size," or "distance") perception. For example, suppose a "length" was specified by a black line presented for e sec in an otherwise homogeneous white visual field (Ganzfeld). If this line were removed and followed $t \mathrm{sec}$ later by an e-sec presentation of a second line, an $O$ could be asked to decide whether the second line was the same length as the first or shorter. If the original line subtended $r$ deg of visual angle while the second subtended $r-m$ deg, the O's ability to perceive the $m \cdot d e g$ change in length should be the same as his ability to perceive the m-deg displacement illustrated in Fig. 2. Note that this argument assumes that the position of the second line is identical except for the $\mathrm{m}$-deg shortening of one end of the line. If the $O$ knew which end of the line might be shortened, he could either compare the original and terminal positions of that end of the line (an absolute judgment) or compare the total length of the line at Time 0 to its length at Time $t$ (a relative judgment). If he did not know which end of the line might be shortened, he would have the same problem as an $O$ in the induced-movement experiment described in the preceding section. He might perceive a shortening of the line without knowing which end had actually been abbreviated. Of course, there are even more complicated procedures where both ends of the line are abbreviated to produce the total $\mathrm{m}$ deg of shortening, or where the line is presented within a visual field containing alternative reference points (such as the edge of a screen). In any case, the importance of such considerations is suggested by the preceding experiment. With adequately controlled experimental conditions, it should be possible to interpret certain "length" judgments using the two models presented in this paper.

\section{THE GENERAL ISSUE OF ABSOLU'TE AND RELATIVE JUDGMENTS}

The theoretical arguments developed here also seem relevant to the perception of other-than-visual-position stimuli. Fur 
example, suppose the stimulus dimension, $\mathrm{L}$, in Fig. 6a denoted the amplitude of $a$ pure tone. If the $O$ were asked to compare Amplitudes $a$ and $b$, his judgments might be more or less independent of $c$, suggesting an absolute judgment process, or critically dependent on $c$, suggesting a relative judgment process. In other words, an $O$ might compare b with his "memory" of a at Time $t$ (as specified by the absolute judgment model); or he might compare the difference, "shift in amplitude," defined at Time $t, c-b$, with his memory of the difference defined earlier at Time $0, c \cdots$ a (as specified in the relative judgment model). In fact, the absolute model was originally applied to an auditory amplitude task (Kinchla \& Smyzer, 1968), in which a and $b$ (Fig. 6a) were clearly audible $1,000-\mathrm{H} 2$ tones and c was "silence" (zero amplitude). Conditions in which the relative judgment model might be applicable would be those in which $\mathrm{c}$ was just barely discriminable from $b$, and where $t$ was quite large, e.g., $t>5$ sec. While no data explicitly bearing on this issue seem to be available in the literature, phenomena of this sort have been demonstrated in this author's laboratory and are the object of current experimentation. An O's ability to discriminate two clearly audible, but barely discriminable, tones presented $5 \mathrm{sec}$ apart against a background of silence is markedly improved if the background amplitude is made similar to, but clearly discriminable from, the other two tones. It seems clear that discrimination improves because the $O$ compares the difference between the first tone and the background with the difference between the second tone and the background. Note that it seems to be the similarity ("proximity") of the background or reference level to the other two amplitudes that is critical, just as it is in the case of relative movement perception. It is easy to devise similar examples of this phenomenon involving other stimulus dimensions, e.g., brigh tness, hue, pitch, spatial position of a tactile stimulus, etc. In any case, the object here is simply to suggest the more general implications of the present analysis of visual movement perception. If two stimulus values seem to be compared independently of their relation to any other "reference" value, one could describe the process as an absolute judgment; if the comparison seems to depend on the difference between each of the two stimulus values and some other "reference" value, the process could be termed a relative judgment. The two models presented here are one way of representing a distinction of this sort.

\section{REFERENCES}

DUNKER, K. Über induzierte Bewegung (ein Beitrag zur Theorie optisch Wohrgenommener). Psychologische Forschung, $1929,12,180-259$

GREEN, D. M., \& SWETS, J. A. Signal detection theory and psychophysics. New York: Wiley, 1966.

KINCHLA, R. A., \& ALLAN, L. G. A theory of visual movement perception. Psychological Review, 1969, 76, 537.558.

KINCHLA, R. A., \& ALLAN, L. G. Visual movement perception: A comparison of sensitivity to vertical and horizontal movement. Perception \& Psychophysics, 1970, $8,399-405$.

KINCHLA, R. A., \& SMYZER, F, A. A diffusion model of perceptual memory. Perception \& Psychophysics, 1967, 2, 219-229.

(Accepred for publication July 6, 1970.) 